\title{
DETERMINING THE ASSOCIATION BETWEEN DERMATOGLYPHICS AND SCHIZOPHRENIA BY USING FINGERPRINT ASYMMETRY MEASURES
}

\author{
JEN-FENG WANG, CHEN-LIANG LIN and CHEN-WEN YEN \\ Department of Mechanical and Electromechanical Engineering \\ National Sun Yat-sen University, Kaohsiung 80424, Taiwan \\ YUNG-HSIEN CHANG*, TENG-YI CHEN and KUAN-PIN SU \\ China Medical University and Hospital, Taichung 40402, Taiwan \\ *vincen@mail.nsysu.edu.tw \\ MARK L. NAGURKA \\ Department of Mechanical Engineering, Marquette University \\ Milwaukee, WI 53201, USA
}

\begin{abstract}
Early detection and intervention strategies for schizophrenia are receiving increasingly more attention. Dermatoglyphic patterns, such as the degree of asymmetry of the fingerprints, have been hypothesized to be indirect measures for early abnormal developmental processes that can lead to later psychiatric disorders such as schizophrenia. However, previous results have been inconsistent in trying to establish the association between dermatoglyphics and schizophrenia. The goal of this work is to try to resolve this problem by borrowing well-developed techniques from the field of fingerprint matching. Two dermatoglyphic asymmetry measures are proposed that draw on the orientation field of homologous fingers. To test the capability of these measures, fingerprint images were acquired digitally from 40 schizophrenic patients and 51 normal individuals. Based on these images, no statistically significant association between conventional dermatoglyphic asymmetry measures and schizophrenia was found. In contrast, the sample means of the proposed measures consistently identified the patient group as having a higher degree of asymmetry than the control group. These results suggest that the proposed measures are promising for detecting the dermatoglyphic patterns that can differentiate the patient and control groups.
\end{abstract}

Keywords: Dermatoglyphic; fingerprint; schizophrenia; fluctuating asymmetry; ridge count; mutual information.

\section{Introduction}

Schizophrenia is hypothesized to be the result of an interaction between genetic factors and insults during embryonic development. ${ }^{22}$ While a full understanding of

*Author for correspondence 
its origins and pathogenic mechanisms remain elusive, schizophrenia is generally considered to be a neurodevelopmental disorder. ${ }^{12}$ This idea is supported by brain imaging studies that have found diverse abnormalities in schizophrenia. ${ }^{4}$ For example, structural brain abnormalities, such as distortion of the corpus callosum and asymmetry of brain, have been recognized on computed tomography (CT) and magnetic resonance imaging (MRI) scans. ${ }^{11,14,17-19,35}$ Studies suggest that schizophrenia may be due to impaired neurodevelopment and might occur before the end of the second gestational trimester.

Dermatoglyphics, the epidermal ridges and patterns of the hand, are established by the end of the second trimester and have been considered as markers of prenatal brain injury. The rationale behind this hypothesis is that epidermal ridges share ectodermal origins with the central nervous system. Specifically, epidermal ridges start to develop in the 11th gestational week and their critical stage of differentiation occurs in fetal months $3-4$, coinciding with a critical phase of brain development. ${ }^{25}$

The morphology of the epidermal ridges is genetically determined but can also be influenced by environmental factors such as a viral infection, radiation, or alcohol and drug abuse that can disturb brain development. ${ }^{2,9}$ However, once they are formed the epidermal ridges remain unchanged. Since both the ridges and the brain are derived from the ectoderm, it seems reasonable to use unusual dermatoglyphic patterns to characterize disturbances to brain development. Preliminary experimental results have successfully demonstrated the association between dermatoglyphics and cerebral structural measures in patients with schizophrenia. ${ }^{31}$

Many studies have tried to establish the direct link between epidermal ridges and schizophrenia using different features to characterize the configuration of epidermal ridges. Fearon et al. ${ }^{10}$ reported more than 70 such studies up to 2001 . These studies are valuable since they considered schizophrenia from a preventive perspective reflecting an important recent trend in neurosciences. ${ }^{7}$ However, as will be reported in the subsequent section, results of these studies were inconsistent.

This paper proposes alternative dermatoglyphic asymmetry measures to differentiate schizophrenic patients from healthy individuals. The measures are adapted from a technique that has been extensively employed in fingerprint matching. The paper is organized as follows. The following section reviews commonly employed finger dermatoglyphic features as well as the results of previous studies. Section 3 illustrates the proposed method. Section 4 presents the experimental results. Discussion and conclusion are given in Sec. 5 .

\section{Previous Work}

Due to the inevitability of developmental errors, no organism is perfectly symmetrical. Individuals have bilateral distribution with different degrees of asymmetry. The degree of asymmetry has thus been considered an indirect measure for developmental instability. ${ }^{36}$ Since dermatoglyphic anomalies are hypothesized to signify disruptions in the second trimester of prenatal development, a critical time period in the etiology of schizophrenia, the possibility that schizophrenic patients tend to have a higher degree of dermatoglyphic asymmetry has been studied extensively. 
Conventional finger dermatoglyphic features that have been used to measure the degree of asymmetry include the following five categories:

(1) Ridge Pattern: Based on the pattern of ridge configuration, several criteria have been proposed to classify fingerprints into different pattern groups. These include the three-pattern system (loop, arch, whorl), ${ }^{8}$ the six-pattern system (plain arch, tended arch, ulnar loop, radial loop, whorl or combined figure), ${ }^{13}$ and the eight-pattern system (plain arch, tended arch, ulnar loop, radial loop, plain whorl, double loop whorl, central pocket loop whorl, and accidental whorl) adopted by the FBI.

(2) FRC and TFRC: The finger ridge count (FRC) is defined as the number of ridges intersected by a line between the triradial points (also called the delta point) to the point of core. As shown in Fig. 1(a), the core is the topmost point of the innermost curving ridge and a triradius is defined as the meeting place of three dermal lines that make angles of approximately $120^{\circ}$ with one another. The total finger ridge count (TFRC) is the sum of FRC for all fingers in one hand. Some fingers may have more than one triradial point, as shown in Fig. 1(b), and this results in multiple ridge counts. To resolve this problem, the largest ridge count is typically chosen as the FRC.

(3) AFRC: The absolute finger ridge count (AFRC) is equal to FRC when the finger has only one triradial point. However, in dealing with multiple triradial points, unlike FRC that uses the largest ridge count, AFRC is chosen as the sum of all ridge counts. The sum of AFRC for all fingers in one hand is denoted as the total absolute finger ridge count (TAFRC) in this work.

(4) AB-TRC: The total $a-b$ ridge count is defined as the number of ridges intersected by a line drawn between the $a$ triradius (at the base of the index finger) and $b$ triradius (at the base of the middle finger) of the palm in each hand, as depicted in Fig. 2.

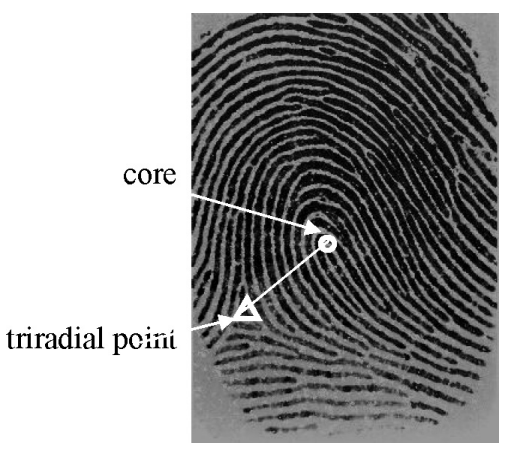

(a)

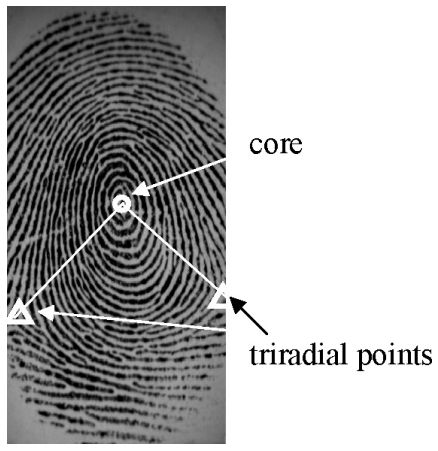

(b)

Fig. 1. (a) A fingerprint with one triradial point. (b) A fingerprint with two triradial points. The ridge count is the number of ridges intersected by a line between the triradial points to the point of core. 


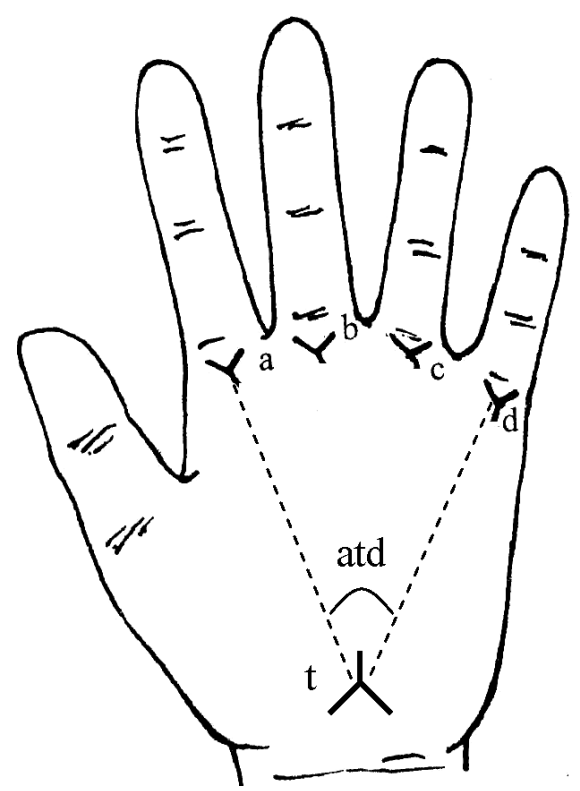

Fig. 2. Example of palmar dermatoglyphics. The straight line crossing the ridges between triradius $a$ and triradius $b$ is the line used for determining the $a-b$ ridge count. Other dermatoglyphic variables $a, b, c, d, t$ and the atd angle are also given in the figure.

(5) atd angle: The atd angle is the angle between two straight lines joining the triradius $a$ and the triradius $d$, under the little finger, with a point $t$, on the lower outer portion of the palm, approximately under the ring finger, as shown in Fig. 2.

A direct approach for characterizing the dermatoglyphic asymmetry is to compare the sample means of these features for homologous fingers. Chok et al. ${ }^{6}$ have summarized the results of many such comparative studies, some of which are not in agreement. For instance, for TFRC, the differences between patient and control groups have been found to be both statistically significant ${ }^{1,6}$ and statistically insignificant. 9,10,27,29,30 Inconsistent results have also been obtained for AB-TRC. Some studies have found that schizophrenic patients tend to have smaller AB$\mathrm{TRC}^{5,9,10}$ whereas other studies have failed to find such a difference. ${ }^{6,28,29}$

Fluctuating asymmetry (FA) represents an alternative criterion for measuring the degree of departure from perfect bilateral symmetry. ${ }^{32}$ In particular, FA quantifies the degree of asymmetry of a human morphological trait by computing the random differences between the right $(\mathrm{R})$ and left $(\mathrm{L})$ sides of the trait. The reason why FA is often used to reflect the developmental instability is that the level of FA is increased when environmental factors or genetic stress during ontogeny degrades the efficiency of normal developmental processes. ${ }^{21}$

By comparing the level of bilateral symmetry for finger dermatoglyphic features, a number of studies have tried to use the FA criterion to investigate if schizophrenic 
patients have a higher degree of asymmetry than normal individuals. Results from these studies are also inconsistent. ${ }^{6}$

As suggested by several authors ${ }^{10,29}$ the lack of consistency in previous work may be due to factors such as diagnostic criteria and ethnicity. Another possibility is that the difference between the degree of finger dermatoglyphic asymmetry for schizophrenic patients and normal individuals is relatively subtle and thus difficult to detect. ${ }^{5}$ In the subsequent section two alternative dermatoglyphic asymmetry measures are posited to detect such potentially subtle differences.

\section{Proposed Approach}

As described in the preceding section, in trying to find the association between dermatoglyphics and schizophrenia inconsistent results have been obtained. Rather than repeat comparative studies using the same dermatoglyphic features, this work focuses on the ridge orientation field (also referred to as the directional field in some literatures) for the homologous fingers. As shown in Fig. 3, the ridge orientation

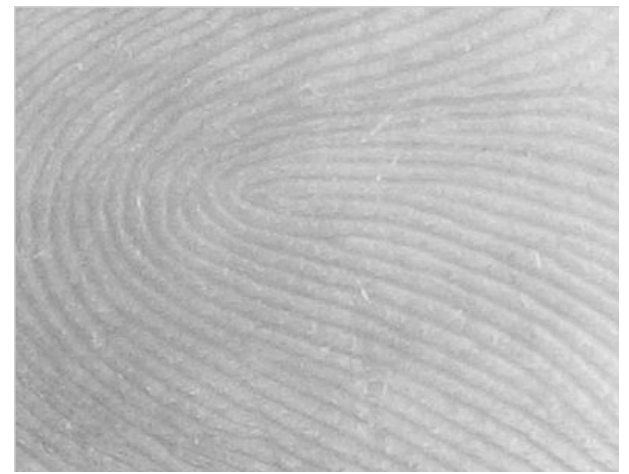

(a) Left finger

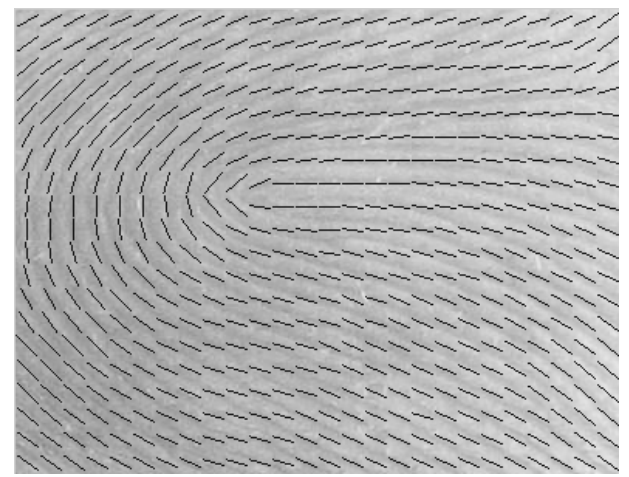

(c) Orientation field of left finger

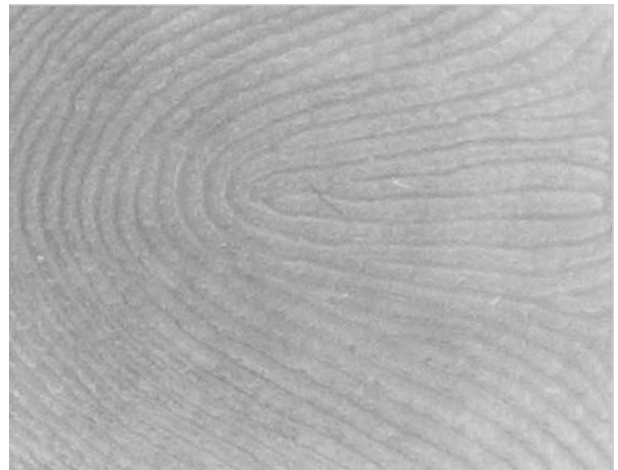

(b) Right finger

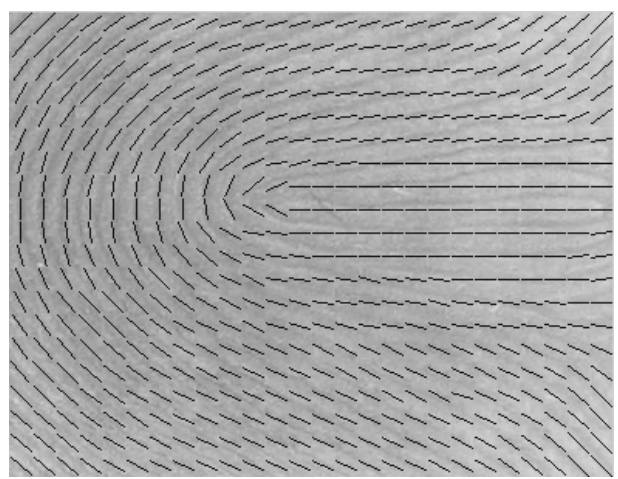

(d) Orientations field of right

Fig. 3. Examples of ridge orientation field for a pair of index fingers. (a) and (b) are the original fingerprint images and (c) and (d) illustrate the orientation field for these two fingers. 
field is a representation of the direction of the ridges throughout the image and thus represents the intrinsic nature of the fingerprint image. With the exception of ridge-pattern, which is only a qualitative measure, traditional dermatoglyphic finger features cannot provide such global information to quantitatively characterize the overall configuration of fingerprints.

\subsection{Acquisition and alignment of finger images}

Fingerprints have traditionally been extracted by creating an inked impression of the fingertip on paper. This acquisition procedure is sensitive to environmental factors and the skin condition, ${ }^{15}$ and consequently many fingerprint images acquired this way are of poor quality. In this work fingerprint images are captured using a digital camera (Canon G3, resolution $2272 \times 1704$ ). Figure 4 shows an index finger image acquired in such a way.

After segmenting the fingers from the background, a preprocessing step for measuring the degree of asymmetry, the contours of homologous fingers are aligned using the maximization of mutual information (MMI) criterion. ${ }^{33}$ By measuring the amount of information that one image contains relative to the other, mutual information (MI) has been applied extensively to image registration problems. ${ }^{20} \mathrm{In}$ particular, given two images $A$ and $B$, the definition of the mutual information $I$ $(A, B)$ of these images is

$$
I(A, B)=H(A)+H(B)-H(A, B)
$$

where $H(A)$ and $H(B)$ denote the entropies of the images $A$ and $B$, respectively. Note that the entropy of an image can be thought of as a dispersion measure for the distribution of the image gray values. $H(A, B)$ is the joint entropy of images $A$ and $B$ and can be used to quantify the dispersion of the joint probability distribution $p(a, b)$ which is the probability of the occurrence for pixels of gray value $a$ in image

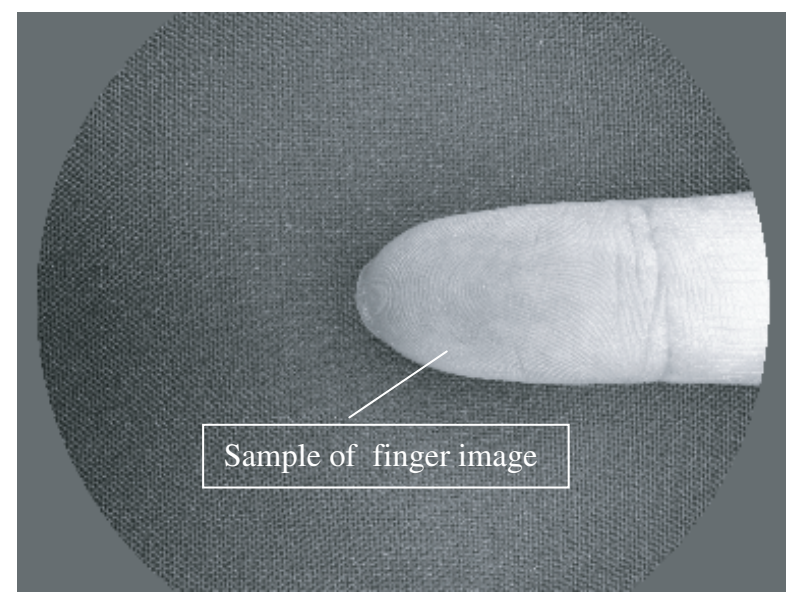

Fig. 4. An example of a digitally captured fingerprint image. 
$A$ and counterpart pixels with gray value $b$ in image $B$. An important property of the joint entropy is that $H(A, B)$ decreases with the strength of the statistical relationship between images $A$ and $B$. Therefore, as images $A$ and $B$ become more similar, the lower the value of $H(A, B)$.

Since the goal is to align the contour of the fingers, finger images are first converted into binary images by setting the background pixels to zeros and the finger pixels to ones. Next, for a pair of homologous fingers, one binary finger image is taken as the reference, and the other binary finger image is aligned by rotating and translating it until an optimal position is found that maximizes the MI. Figure 6 shows the contours of the homologous fingers of Fig. 5 before and after alignment.

\subsection{Computing orientation field for the region of interest}

After aligning the finger images, the next step is to determine the region of interest (ROI) for each finger. As illustrated in Fig. 7, the ROI is chosen as a $1.43 \times 1.10 \mathrm{~cm}^{2}$ rectangular whose center is located at the centroid of the fingertip area.

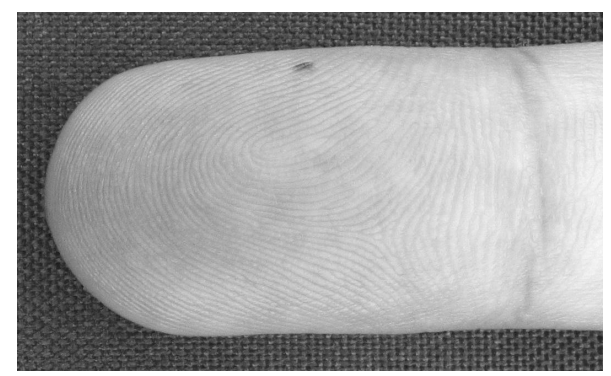

(a) Left finger

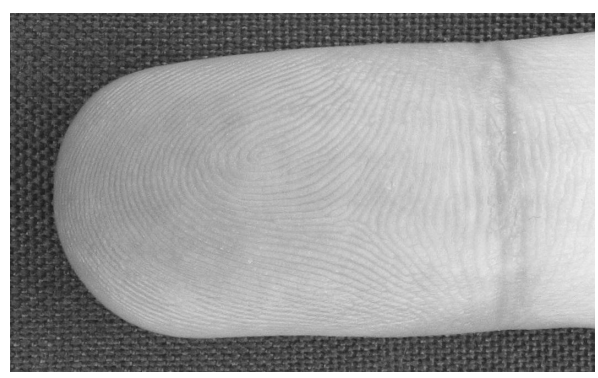

(b) Right finger

Fig. 5. Examples of a pair of homologous finger images before the operation of alignment. The finger image shown in (a) is set as the reference and finger image of (b) is to be adjusted by the MMI criterion.

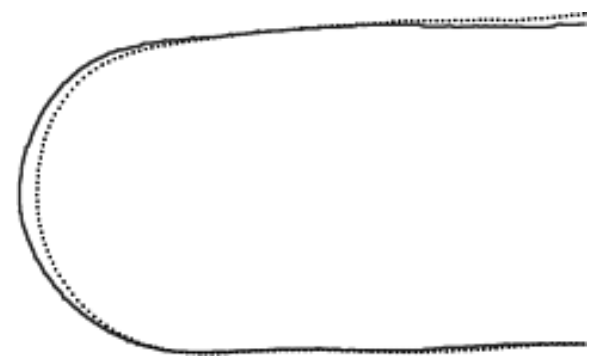

(a) Before alignment

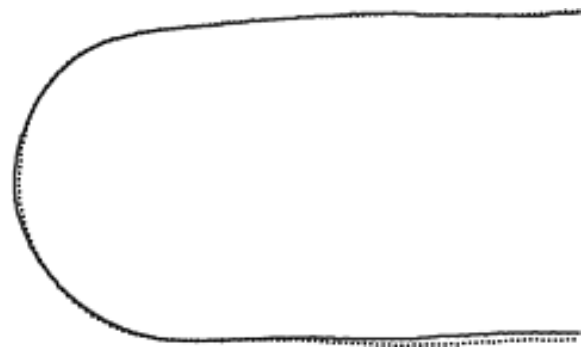

(b) After alignment

Fig. 6. An illustration of the effect of alignment for the pair of homologous fingers shown in Fig. 5. (a) Contours before alignment. (b) Contours after alignment. Note that the dotted line represents the contour of left finger and solid line is the contour of the right finger. 


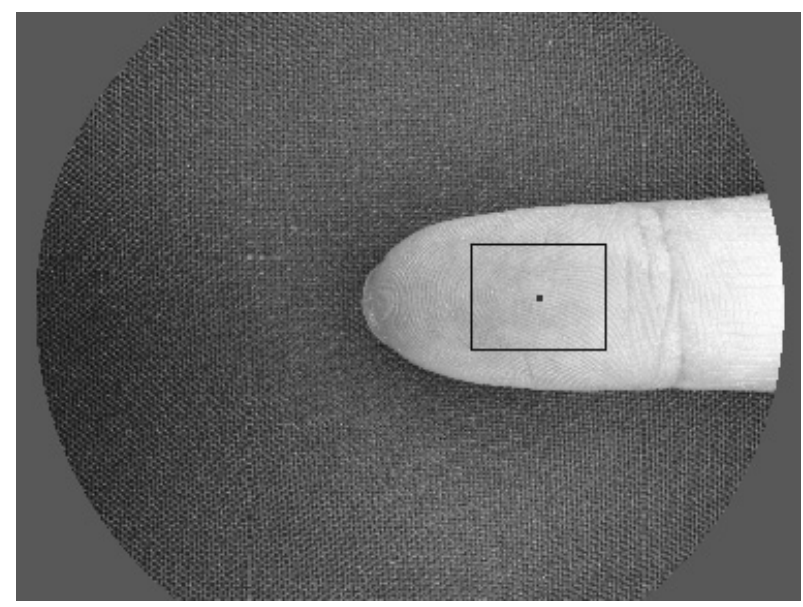

Fig. 7. The region of interest (ROI) used in this work for the fingerprint asymmetry study. The ROI is a $1.43 \times 1.10 \mathrm{~cm}^{2}$ rectangular whose center is chosen as the centroid of the fingertip.

The following procedure, which is adapted from a method proposed by Rao, ${ }^{26}$ is used to compute the orientation field for the selected ROI:

- Divide the ROI into a number nonoverlapping blocks of size $15 \times 15$.

- Compute the horizontal and vertical gradients using the Sobel operator for each pixel of the ROI.

- Calculate the orientation angle for each block using the gradients determined in the previous step.

- Smooth the computed orientation field by low-pass filtering.

Due to the presence of smudges, noise and breaks, etc. an orientation field error is often inevitable. To accurately compute the proposed asymmetry measures, the orientation field of every tested fingerprint image used here has been manually inspected and corrected when necessary.

\subsection{Proposed fingerprint asymmetry measures}

The block structure used in the previous subsection for orientation field computation is the basis for quantifying the degree of asymmetry for a pair of homologous fingers. The first fingerprint asymmetry measure proposed is the difference measure defined as

$$
\text { Difference Measure }=\frac{1}{N} \sum_{i=1}^{N}\left|p_{i}-t_{i}\right|
$$

where $p_{i}$ and $t_{i}$ represent the direction angle of the $i$ th block of the right and left hand fingers, respectively, and $N$ is the number of blocks used for the orientation field computation. 
The second asymmetry measure used in this work is called the correlation measure. It can be computed from

$$
\text { Correlation Measure }=\frac{\sum_{i=1}^{N}\left(p_{i}-\bar{p}\right)\left(t_{i}-\bar{t}\right)}{\sqrt{\sum_{i=1}^{N}\left(p_{i}-\bar{p}\right)^{2}} \sqrt{\sum_{i=1}^{N}\left(t_{i}-\bar{t}\right)^{2}}}
$$

where $\bar{p}$ and $\bar{t}$ are the sample means of the orientation angles for the fingers of the right and left hands, respectively. This measure is often used in signal processing applications to characterize the degree of similarity between two signals.

\section{Experimental Results}

Fingerprint images were acquired from 40 patients diagnosed with schizophrenia from the Jing-Ho Mental Hospital (Changhua, Taiwan) and the China Medical University Hospital (Taichung, Taiwan). Fingerprint images were also acquired from 51 control participants. For the control group, 49 participants were students at Sun Yat-sen University (Kaohisung, Taiwan) and the remaining two were volunteers from the China Medical University Hospital. All participants were of Han nationality and thus belong to the same ethnic group. In addition, to eliminate the potential effect of sexual differences, all participants were chosen to be males.

Fingerprint images were acquired by a digital camera, and for the sake of reliability, five consecutive shots were taken for each finger. In this work thumbs were excluded from the experimental study. (Taking accurate images of thumbs was found to be much more time consuming than other fingers.)

With the availability of five images for each finger, every image of a right-hand finger was compared with every image of the homologous finger of the left-hand. Among the 25 combinations, the one that yielded the smallest asymmetry measure value was adopted.

Mean values were assessed using a two-sided independent samples $t$-test. The test results were considered significant for $P$-values $<0.01$. Each of the tested asymmetry measures was found in five comparative studies for the index, middle, ring and little fingers as well as the overall result (sum or average) for the four fingers. Since triradial points could not be found in some of our fingerprint images, the number of samples for each comparison often differs from the number of participants.

The first part of the experiment tests the hypothesis that the control group has larger FRC or AFRC than the patient group. The results, summarized in Tables 1 and 2 , indicate that there are no significant differences between the groups for FRC or AFRC.

The second part of the experiment investigates the degree of bilateral symmetry. In particular, the question is if the patient group has a higher level of FRC or AFRC fluctuating asymmetry in comparison to the normal group. An FA measure adopted in several previous fingerprint asymmetry studies ${ }^{1,6,21,30,34}$ is used here. It is the 
Table 1. Summary of the results for FRC and TFRC.

\begin{tabular}{lcccccc}
\hline & & \multicolumn{2}{c}{ Controls } & & \multicolumn{2}{c}{ Patients } \\
Fingers & Numbers & Mean \pm Std. & Numbers & & Mean \pm Std. & $P$-Value \\
\hline FRC & & & & & \\
$\quad$ Left Hand & & & & & \\
$\quad$ Index & 45 & $13.58 \pm 5.34$ & 34 & & $13.91 \pm 6.46$ & 0.808 \\
$\quad$ Middle & 46 & $14.20 \pm 4.73$ & 33 & & $14.12 \pm 4.47$ & 0.943 \\
$\quad$ Ring & 45 & $16.49 \pm 3.82$ & 31 & & $16.03 \pm 4.81$ & 0.661 \\
$\quad$ Little & 48 & $14.02 \pm 3.46$ & 37 & & $14.19 \pm 5.00$ & 0.862 \\
Right Hand & & & & & \\
$\quad$ Index & 43 & $13.47 \pm 4.66$ & 31 & & $13.19 \pm 5.67$ & 0.828 \\
$\quad$ Middle & 45 & $14.00 \pm 4.15$ & 33 & & $13.03 \pm 4.96$ & 0.365 \\
$\quad$ Ring & 46 & $15.96 \pm 3.32$ & 26 & & $15.69 \pm 5.75$ & 0.831 \\
$\quad$ Little & 46 & $13.89 \pm 3.84$ & 35 & & $14.89 \pm 5.16$ & 0.342 \\
TFRC & 32 & $107.84 \pm 24.46$ & 16 & & $97.13 \pm 38.37$ & 0.320 \\
\hline
\end{tabular}

Table 2. Summary of the results for AFRC and TAFRC.

\begin{tabular}{lcclccc}
\hline & \multicolumn{2}{c}{ Controls } & & \multicolumn{2}{c}{ Patients } & \\
\cline { 2 - 3 } Fingers & Numbers & Mean \pm Std. & & Numbers & Mean \pm Std. & $P$-Value \\
\hline AFRC & & & & & & \\
$\quad$ Left Hand & & & & & & \\
$\quad$ Index & 45 & $19.56 \pm 11.06$ & & 34 & $20.35 \pm 12.73$ & 0.772 \\
$\quad$ Middle & 46 & $19.43 \pm 11.21$ & & 33 & $19.73 \pm 10.35$ & 0.905 \\
$\quad$ Ring & 45 & $24.16 \pm 9.93$ & & 31 & $26.45 \pm 12.03$ & 0.384 \\
Little & 48 & $17.42 \pm 7.90$ & & 37 & $19.22 \pm 9.66$ & 0.360 \\
Right Hand & & & & & & \\
$\quad$ Index & 43 & $19.67 \pm 10.88$ & & 31 & $18.29 \pm 11.15$ & 0.597 \\
Middle & 45 & $19.44 \pm 10.70$ & & 33 & $16.67 \pm 9.75$ & 0.237 \\
$\quad$ Ring & 46 & $23.39 \pm 8.92$ & & 26 & $24.73 \pm 11.88$ & 0.619 \\
$\quad$ Little & 46 & $17.24 \pm 7.98$ & & 35 & $20.69 \pm 10.32$ & 0.106 \\
TAFRC & 32 & $142.06 \pm 57.54$ & & 16 & $128.81 \pm 67.12$ & 0.505 \\
\hline
\end{tabular}

first FA asymmetry measure used in this work,

$$
F A_{1}=\frac{1}{M} \sum_{i=1}^{M}\left|L_{i}-R_{i}\right|,
$$

where $R_{i}$ and $L_{i}$ are the values of the tested feature of the $i$ th finger of the right and left hands, respectively, and $M$ is the number of fingers used for comparison.

The second FA measure used here is

$$
F A_{2}=\frac{1}{M} \sum_{i=1}^{M} \frac{\left|L_{i}-R_{i}\right|}{\left|L_{i}+R_{i}\right|} .
$$

This criterion has also been used in previous fingerprint asymmetry studies. ${ }^{23,27,29}$ The results for the two tested FA asymmetry measures are shown in Tables 3 and 4, and indicate that no statistically significant difference can be found. 
Table 3. Summary of the results for the fluctuating dermatoglyphic asymmetry measure $F A_{1}$.

\begin{tabular}{|c|c|c|c|c|c|c|}
\hline \multirow[b]{2}{*}{ Ridge Count Measure } & \multirow[b]{2}{*}{ Finger } & \multicolumn{2}{|c|}{ Controls } & \multicolumn{2}{|c|}{ Patients } & \multirow[b]{2}{*}{$P$-Value } \\
\hline & & Numbers & Mean \pm Std. & Numbers & Mean \pm Std. & \\
\hline \multirow{5}{*}{ FRC } & Index & 40 & $2.45 \pm 2.43$ & 28 & $2.11 \pm 1.55$ & 0.480 \\
\hline & Middle & 42 & $2.45 \pm 1.73$ & 28 & $2.86 \pm 2.32$ & 0.434 \\
\hline & Ring & 42 & $2.05 \pm 1.77$ & 25 & $2.20 \pm 1.71$ & 0.729 \\
\hline & Little & 46 & $2.40 \pm 1.88$ & 34 & $2.18 \pm 1.53$ & 0.870 \\
\hline & Total & 32 & $2.23 \pm 0.97$ & 16 & $2.03 \pm 1.15$ & 0.564 \\
\hline \multirow{5}{*}{ AFRC } & Index & 40 & $3.95 \pm 4.22$ & 28 & $3.57 \pm 4.62$ & 0.732 \\
\hline & Middle & 42 & $5.33 \pm 5.64$ & 28 & $4.96 \pm 4.99$ & 0.775 \\
\hline & Ring & 42 & $5.07 \pm 4.93$ & 25 & $2.68 \pm 2.56$ & 0.011 \\
\hline & Little & 46 & $3.41 \pm 3.05$ & 34 & $3.97 \pm 4.06$ & 0.504 \\
\hline & Total & 32 & $4.02 \pm 2.36$ & 16 & $3.33 \pm 1.74$ & 0.261 \\
\hline
\end{tabular}

Table 4. Summary of the results for the fluctuating dermatoglyphic asymmetry measure $F A_{2}$.

\begin{tabular}{|c|c|c|c|c|c|c|}
\hline \multirow[b]{2}{*}{ Ridge Count Measure } & \multirow[b]{2}{*}{ Finger } & \multicolumn{2}{|c|}{ Controls } & \multicolumn{2}{|c|}{ Patients } & \multirow[b]{2}{*}{$P$-Value } \\
\hline & & Numbers & Mean \pm Std. & Numbers & Mean \pm Std. & \\
\hline \multirow{5}{*}{ FRC } & Index & 40 & $0.121 \pm 0.155$ & 28 & $0.149 \pm 0.225$ & 0.563 \\
\hline & Middle & 42 & $0.099 \pm 0.090$ & 28 & $0.145 \pm 0.194$ & 0.246 \\
\hline & Ring & 42 & $0.066 \pm 0.056$ & 25 & $0.123 \pm 0.220$ & 0.216 \\
\hline & Little & 46 & $0.095 \pm 0.104$ & 34 & $0.092 \pm 0.095$ & 0.892 \\
\hline & Total & 32 & $0.090 \pm 0.051$ & 16 & $0.122 \pm 0.125$ & 0.339 \\
\hline \multirow{5}{*}{ AFRC } & Index & 40 & $0.143 \pm 0.174$ & 28 & $0.170 \pm 0.235$ & 0.607 \\
\hline & Middle & 42 & $0.147 \pm 0.131$ & 28 & $0.184 \pm 0.202$ & 0.399 \\
\hline & Ring & 42 & $0.119 \pm 0.116$ & 25 & $0.116 \pm 0.223$ & 0.956 \\
\hline & Little & 46 & $0.117 \pm 0.114$ & 34 & $0.118 \pm 0.110$ & 0.954 \\
\hline & Total & 32 & $0.124 \pm 0.074$ & 16 & $0.139 \pm 0.117$ & 0.647 \\
\hline
\end{tabular}

Table 5. Summary of the results for the proposed orientation difference measure.

\begin{tabular}{lccc}
\hline & \multicolumn{2}{c}{$\begin{array}{c}\text { Orientation Difference } \\
\text { (Mean } \pm \text { Standard Deviation })\end{array}$} & \\
\cline { 2 - 3 } Finger & Controls & Patients & $P$-Value \\
\hline Index & $12.78 \pm 5.30$ & $13.62 \pm 7.04$ & 0.5333 \\
Middle & $10.82 \pm 4.23$ & $11.13 \pm 4.65$ & 0.7405 \\
Ring & $10.32 \pm 4.16$ & $10.59 \pm 4.01$ & 0.7494 \\
Little & $8.87 \pm 3.89$ & $11.30 \pm 4.52$ & 0.0085 \\
Average & $10.70 \pm 2.87$ & $11.66 \pm 3.20$ & 0.1206 \\
\hline
\end{tabular}

The third part of the experiment performs comparisons of the proposed asymmetry measures and the results are summarized in Tables 5 and 6 . For the difference measure, the results of Table 5 show that the sample means of the schizophrenic group are larger than those of the control group in all five experiments. In addition, the result for the little finger has achieved statistical significance 
Table 6. Summary of the results for the proposed correlation measure.

\begin{tabular}{|c|c|c|c|}
\hline \multirow[b]{2}{*}{ Finger } & \multicolumn{2}{|c|}{$\begin{array}{c}\text { Correlation } \\
(\text { Mean } \pm \text { Standard Deviation })\end{array}$} & \multirow[b]{2}{*}{$P$-Value } \\
\hline & Controls & Patients & \\
\hline Index & $0.826 \pm 0.096$ & $0.785 \pm 0.121$ & 0.0809 \\
\hline Middle & $0.840 \pm 0.087$ & $0.802 \pm 0.104$ & 0.0698 \\
\hline Ring & $0.848 \pm 0.086$ & $0.813 \pm 0.115$ & 0.1110 \\
\hline Little & $0.869 \pm 0.073$ & $0.820 \pm 0.120$ & 0.0256 \\
\hline Average & $0.846 \pm 0.057$ & $0.805 \pm 0.071$ & 0.0038 \\
\hline
\end{tabular}

$(P$-value $=0.0085)$. These results suggest that the patient group tends to have a higher degree of asymmetry than the control group.

For the proposed correlation measure, the results presented in Table 6 confirm that the sample means of the patient group are all smaller than those of the normal group in the five tests. Statistical significance has also been reached for the average of the correlation measure of the four tested fingers $(P$-value $=0.0038)$. The results obtained using the proposed correlation measure agree with the results obtained by the difference measure in that they also suggest that the patient group tends to have a higher degree of asymmetry than the control group.

\section{Discussion and Conclusion}

Schizophrenia is considered to be associated with altered prenatal neurodevelopment. Since dermatoglyphic patterns are formed at the second trimester of prenatal development, a time period that appears to be etiologically relevant to the development of schizophrenia, unusual dermatoglyphic patterns have been hypothesized to be proxy markers of altered early development in psychosis. To test this hypothesized association, this work compares the degree of finger dermatoglyphic asymmetry for schizophrenic and normal groups. In addition to using conventional asymmetry measures, this work proposes two alternative measures based on the orientation field of the fingerprints. For our tested sample, the conventional dermatoglyphic measures were not able to provide statistically significant results that can be used to differentiate the patient and the control groups. Therefore, the association between schizophrenia and the conventional dermatoglyphic asymmetry measures cannot be established in this study.

In contrast, as demonstrated by the results of Tables 5 and 6 , the sample means of the proposed difference (correlation) measure of the patient group are all larger (smaller) than those of the control group. In addition among the ten tests of Tables 5 and 6, two have achieved statistical significance. The limited success of the proposed measures may be due to the inadequate statistical power of our tests since the number of participants in our experimental studies is relatively small. Nevertheless, compared with the conventional measures, results obtained 
using the proposed measures have provided evidence for an association between unusual dermatoglyphic characteristics and genetic vulnerability to schizophrenia.

The proposed approach extracts information from the orientation field, which characterizes the global configuration of a fingerprint by describing the local directions of the ridge lines. Experimental results have shown that the proposed measures can identify the difference between the patient and normal groups when the conventional dermatoglyphic measures fail to detect any between group differences. It may be valuable to repeat the experiments using the proposed measure for the studies that were unsuccessful in finding an association between finger dermatoglyphics and schizophrenia.

\section{References}

1. M. T. Avila, J. Sherr, L. E. Valentine, T. A. Blaxton and G. K. Thaker, Neurodevelopmental interactions conferring risk for schizophrenia: a study of dermatoglophic markers in patients and relatives, Schizophr. Bull. 29 (2003) 595-605.

2. W. J. Babler, Embryonic development of epidermal ridges and their configurations, in Dermatoglyphics: Science in Transition, Vol. 27, eds. C. C. Plato, R. M. Garruto and B. A. Schaumman (Wiley-Liss, New York, 1991), pp. 95-112.

3. A. M. Bazen and S. H. Gerez, Systematic methods for the computation of the directional fields and singular points of fingerprints, IEEE Trans. Patt. Anal. Mach. Intell. 24(7) (2002) 905-919.

4. B. Bogerts, Recent advances in the neuropathology of schizophrenia, Schizophr. Bull. 19 (1993) 431-445.

5. E. Bramon, M. Walshe, C. McDonald, B. Martín, T. Toulopoulou, H. Wickham, J. van Os, P. Fearon, P. C. Sham, L. Fananás and R. M. Murray, Dermatoglyphics and schizophrenia: a meta-analysis and investigation of the impact of obstetric complications upon a-b ridge count, Schizophr. Res. 75 (2005) 399-404.

6. J. T. Chok, T. R. Kwapil and A. Scheuermann, Dermatoglyphic anomalies in psychometrically identified schizotypic young adults, Schizophr. Res. 72 (2005) 205-214.

7. M. T. Compton, Considering schizophrenia from a prevention perspective, Am. J. Prev. Med. 26 (2004) 178-185.

8. H. Cummins and C. Midlo, Fingerprints, Palms and Soles (Blakiston, Philadelphia, 1943).

9. L. Fañanas, J. van Os, C. Hoyos, J. McGrath, C. S. Mellor and R. Murray, Dermatoglyphic a-b ridge count as a possible marker for developmental disturbance in schizophrenia: replication in two samples, Schizophr. Res. 20 (1996) 307-314.

10. P. Fearon, A. Lane, M. Airie, J. Scannell, A. McGowan, M. Bynre, M. Cannon, D. Cotter, P. Murphy, B. Cassidy, J. Waddington, C. Larkin and E. O'Callaghan, Is reduced dermatoglyphic a-b ridge count a reliable marker of developmental impairment in schizophrenia, Schizophr. Res. 50 (2001) 151-157.

11. J. Foong, M. Maier, C. A. Clark, G. J. Barker, D. H. Miller and M. A. Ron, Neuropathological abnormalities of the corpus callosum in schizophrenia: a diffusion tensor imaging study, J. Neurol. Neurosurg. Psychiatry 68 (2000) 242-244.

12. P. J. Harrison, The neuropathology of schizophrenia: a critical review of the data and their interpretation, Brain 122 (1999) 593-624.

13. E. Henry, Classification and Uses of Finger Prints, 8th edn. (H.M. Stationary Office, London, 1937). 
14. B. Ismail, E. Cantor-Graae and T. F. McNeil, Minor physical anomalies in schizophrenic patients and their siblings, Am. J. Psychiatry 155 (1998) 1695-1702.

15. L. C. Jain, U. Halici, I. Hayashi, S. B. Lee, S. Tsutsui, Intelligent Biometric Techniques in Fingerprint and Face Recognition (CRC Press, Boca Raton, FL, 1999).

16. A. K. Jain, L. Hong, S. Pankanti and R. Bolle, An identity-authentication system by using fingerprints, Proc. IEEE 85 (1997) 1365-1388.

17. B. Kirkpatrick, D. Litman, J. W. Kim, K. Vladar, A. Breier and R. W. Buchanan, Failure of fusion of the septum pellucidum and heterogeneity of schizophrenia, J. Nerv. Ment. Dis. 185 (1997) 639-641.

18. J. S. Kwon, M. E. Shenton, Y. Hirayasu, D. F. Salisbury, I. A. Fischer, C. C. Dickey, D. Yurgelun-Todd, M. Tohen, R. Kikinis, F. A. Jolesz and R. W. McCarley, MRI study of cavum septi pellucidi in schizophrenia, affective disorder, and schizotypal personality disorder, Am. J. Psychiatry 155 (1998) 509-515.

19. A. Lane, A. Kinsella, P. Murphy, M. Byrne, J. Keenan, K. Colgan, B. Cassidy, N. Sheppard, R. Horgan, J. L. Waddington, C. Larkin and E. O'Callaghan, The anthropometric assessment of dysmorphic features in schizophrenia as an index of its developmental origins, Psychol. Med. 27 (1997) 1155-1164.

20. F. Maes, D. Vandermeulen and P. Suetens, Medical image registration using mutual information, Proc. IEEE 91 (2003) 1699-1722.

21. T. A. Markow and K. Wandler, Fluctuating dermatoglyphic asymmetry and the genetics of liability to schizophrenia, Psychiatry Res. 19 (1986) 323-328.

22. G. Michael, M. Richard and G. John, Psychiatry, 2nd edn. (Oxford University Press, New York, 1999).

23. A. R. Palmer and C. Strobeck, Fluctuating asymmetry: measurement, analysis, patterns, Ann. Rev. Ecol. Syst. 17 (1986) 391-421.

24. J. Qi, S. Yang and Y. Wang, Fingerprint matching combining the global orientation field with minutia, Patt. Recogn. Lett. 26 (2005) 2424-2430.

25. P. Rakic, Specification of cerebral cortical areas, Science, 241 (1988) 170-176.

26. A. R. Rao, A Taxonomy for Texture Description and Identification (Springer-Verlag, New York, 1990).

27. J. L. Reilly, P. T. Murphy, M. Bynre, C. Larkin, M. Gill, E. O'Callaghan and A. Lane, Dermatoglyphic fluctuating aasymmetry and atypical handedness in schizophrenia, Schizophr. Res. 50 (2001) 159-168.

28. A. Rosa, M. J. Cuesta, V. Peralta, A. Zarzuela, F. Serrano, A. Martínez-Larrea and L. Fananás, Dermatoglyphic anomalies and neurocognitive deficits in sibling pairs discordant for schizophrenia spectrum disorders, Psychiatry Res. 137 (2005) 215-221.

29. S. Saha, D. Loesch, D. Chant, J. Welham, O. El-Saadi, Fañanás L., B. Mowry and J. McGrath, Directional and fluctuating asymmetry in finger and a-b ridge counts in psychosis: a case-control study, BMC Psychiatry 3 (2003) 1-9.

30. C. J. van Oel, W. F. C. Baaré, H. E. H. Pol, J. Haag, J. Balazs, A. Dingemans, R. S. Kahn and M. M. Sitskoorn, Differentiating between low and high susceptibility to schizophrenia in twins: the significance of dermatoglyphic indices in relation to other determinants of brain development, Schizophr. Res. 52 (2001) 181-193.

31. J. van Os, P. W. R. Woodruff, L. Fañanas, F. Ahmad, N. Shuriquie, R. Howard and R. M. Murray, Association between cerebral structural abnormalities and dermatoglyphic ridge counts in schizophrenia, Compr. Psych. 41 (2000) 380-384.

32. L. Van Valen, A study of fluctuating asymmetry, Evolution 16 (1962) 125-142.

33. P. Viola, Alignment by maximization of mutual information (Ph.D. thesis) (Massachusetts Institute of Technology, 1995). 
34. D. D. Weinstein, D. Diforio, J. Schiffman, E. Walker and R. Bonsall, Minor physical anomalies, dermatoglyuphic asymmetries and cortisol levels in adolescents with schizotypal personality disorder, Am. J. Psychiatr. 156 (1999) 617-623.

35. P. W. Woodruff, I. C. McManus and A. S. David, Meta-analysis of corpus callosum size in schizophrenia, J. Neurol. Neurosurg. Psychiatry 58 (1995) 457-461.

36. R. A. Yeo and S. W. Gangestad, Developmental instability and phenotypic variation in neural organization, in The Other Side of the Error Term: Aging and Development as Model Systems in Cognitive Neuroscience, ed. N. Raz (St. Elsevier Science, New York, 1998), pp. 1-51.

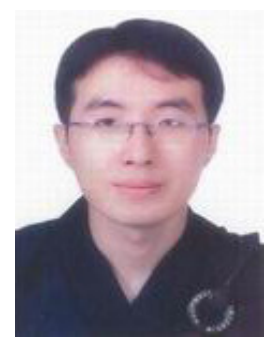

Jen-Feng Wang received his B.E. and M.E. degrees in mechanical engineering (ME) and mechanical and electro-mechanical engineering (MEM) from National Sun Yat-Sen University in 2001 and 2003, respectively. He is currently a Ph.D. student at National Sun Yat-Sen University.

His research interests include the application of image processing, and neural networks to pattern recognition problems.

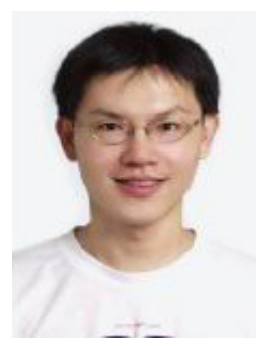

Chen-Liang Lin received the B.E. and M.E. degrees in mechanical engineering (ME) from National Sun YatSen University, in 1999 and 2001, respectively, where he is currently working toward the Ph.D. degree.

His research interests include artificial neural network, pattern recognition and medical signal processing.

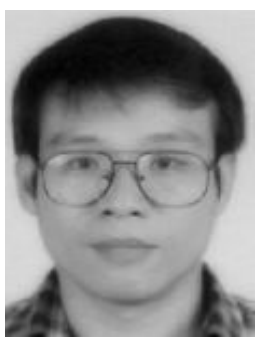

Chen-Wen Yen received his M.E. and Ph.D. degrees in mechanical engineering from Carnegie-Mellon University in 1986 and 1989, respectively. Following graduation, he joined the faculty in the Department of Mechanical Engineering at Sun Yat-Sen University.

The focus of his research work is on the application of neural networks for medical imaging applications.

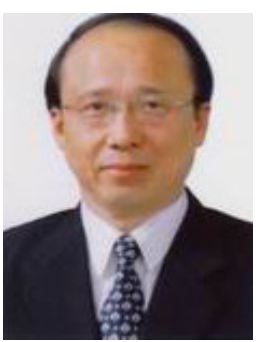

Yung-Hsien Chang is a consultative physician in China Medical University Hospital, Professor in Graduate Institute of Integrative Medicine and vice president at China Medical University.

His fields of specialty include Chinese medicine, acupuncture, rehabilitation, orthopedics, and integrative medicine. 


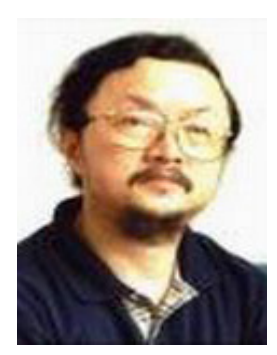

Teng-Yi Chen received his M.D. degree from the College of Chinese Medicine in 1980. He is now a superintendent of CHING-HO hospital in Taichung, Taiwan.

His research works include psychology and schizophrenia.

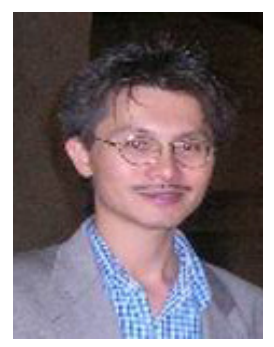

Kuan-Pin $\mathbf{S u}$ is the Chief and Assistant Professor at the Department of General Psychiatry and the Director of Mind-Body Interface Research Center of China Medical University Hospital in Taichung, Taiwan. In 2005, he began to pursue his PhD program in the section of Stress, Psychiatry and Immunology Laboratory at the Institute of Psychiatry, King's College London to study the mechanism of cytokine-induced depression.

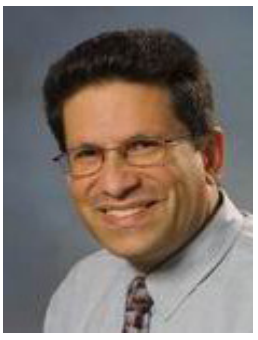

Mark L. Nagurka received the B.S. and M.S. degrees in mechanical engineering and applied mechanics from the University of Pennsylvania, Philadelphia, and the Ph.D. degree in mechanical engineering from Massachusetts Institute of Technology, Cambridge. He then joined the Department of Mechanical and Industrial Engineering at Marquette University, Milwaukee, WI.

His research interests include automation, mechatronics, control design and biomechanics. 